\title{
Pulsars as Astrophysical Laboratories for Nuclear and Particle Physics
}

\author{
F. Weber ${ }^{1}$ R. Negreiros,${ }^{1}$ P. Rosenfield,${ }^{1}$ \\ M. Stejner ${ }^{1,2}$ \\ ${ }^{1}$ Department of Physics, San Diego State, University, \\ 5500 Campanile Drive, San Diego, California 92182, USA \\ ${ }^{2}$ Department of Physics and Astronomy, University of Aarhus, \\ Ny Munkegade, DK-8000 Aarhus C, Denmark
}

February 4, 2008

\begin{abstract}
A forefront area of research concerns the exploration of the properties of hadronic matter under extreme conditions of temperature and density, and the determination of the equation of state-the relation between pressure, temperature and density-of such matter. Experimentally, relativistic heavy-ion collision experiments enable physicists to cast a brief glance at hot and ultra-dense matter for times as little as about $10^{-22}$ seconds. Complementary to this, the matter that exists in the cores of neutron stars, observed as radio pulsars, X-ray pulsars, and magnetars, is at low temperatures but compressed permanently to ultra-high densities that may be more than an order of magnitude higher than the density of atomic nuclei. This makes pulsars superb astrophysical laboratories for medium and high-energy nuclear physics, as discussed in this paper.
\end{abstract}

\section{Introduction}

Exploring the properties of hadronic matter under extreme conditions of temperature and density has become a forefront area of modern physics, both theoretically and experimentally. On the earth, heavy ion experiments enable physicists to cast a brief glance at such matter for times as little as about $10^{-22}$ seconds. On the other hand, it is well known that galaxies like our Milky Way contain up to $10^{8}$ to $10^{9}$ neutron stars, which are observed as pulsars (rotating neutron stars). Such objects contain ultra-dense hadronic matter as a permanent component of matter in their centers. Radio telescopes, X-ray satellites-and soon the latest generation of gravitational-wave detectors-provide physicists and astronomers with an unprecedented wealth of high-quality data on such objects and thus serve as the observational windows on the inner workings of pulsars. This feature makes pulsars superb astrophysical laboratories for medium and high-energy nuclear physics (plus other fields of physics) [1, 2, 3, 4, 4, 6, 6, 7, 8, Some of the key questions that can be addressed by studying the properties of pulsars are (see Fig. 11):

- What are the fundamental building blocks of cold ultra-dense matter? Specifically, does "exotic" matter exist in the cores of pulsars, such as boson condensates, color-superconducting quark matter, and multi-quark states? 


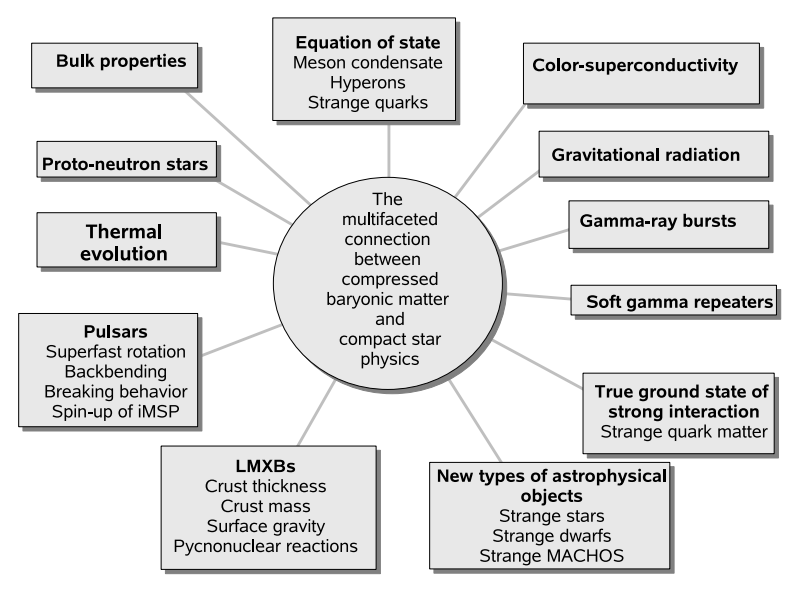

Figure 1: The multifaceted connection between compressed baryonic matter matter and pulsar physics [6].

- Are there pulsar observables that could signal the existence of exotica in their cores?

- Are there rotationally-driven (accretion-driven) phase transitions in pulsars?

- How does color-superconducting quark matter alter the properties of pulsars?

- What is the true ground state of the strong interaction? Is it ordinary nuclear matter (i.e. atomic nuclei) or a color-neutral collection of up, down, and strange quarks (so-called strange quark matter)?

- What are the distinguishing features of pulsars made of strange quark matter rather than ordinary hadronic matter?

- What are the properties of matter subjected to ultra-high density radiation fields, ultra-high magnetic fields, ultra-high electric fields? How do such fields alter the properties of pulsars?

- What are the key nuclear (heavy ion) reactions in the non-equilibrium crusts of accreting X-ray pulsars?

- How strongly do pycnonuclear reactions in the crusts of accreting neutron stars alter the thermal evolution of such objects?

- Do gravitational-radiation reaction driven instabilities limit the spins of pulsars?

- What is the shell structure for very neutron rich nuclei in the crusts of pulsars? Do $\mathrm{N}=50$ and $\mathrm{N}=82$ remain magic numbers?

Before we discuss some of these issues in more detail, we review some of the key properties of neutron stars. The first property concerns the masses of neutron stars, which range theoretically from around $0.1 M_{\odot}\left(\right.$ where $M_{\odot}=2 \times 10^{33} \mathrm{~g}$ is the mass of the sun) to about $3 M_{\odot}$. Matter in the centers of neutron stars possess densities ranging from a few times $n_{0}$ to an order of magnitude higher. Here $n_{0}=0.15$ nucleons $/ \mathrm{fm}^{3}$ denotes the baryon number density of normal nuclear matter, which corresponds to a mass density of $2.5 \times 10^{14} \mathrm{~g} / \mathrm{cm}^{3}$. The number of baryons forming a neutron star is of the order of $10^{57}$. Rotating neutron stars are called pulsars. Three distinct classes of pulsars are currently known. These are (1) rotation-powered pulsars, where the loss of rotational energy of the star powers 


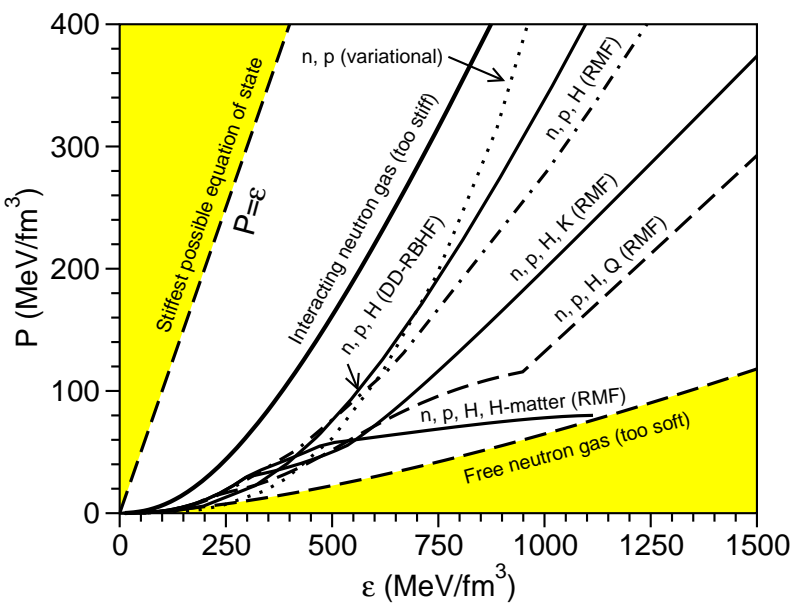

Figure 2: Models for the EoS (pressure versus energy density) of neutron star matter [6]. The notation is as follows: $\mathrm{RMF}=$ relativistic mean-field model; DD-RBHF=density dependent relativistic Brueckner-Hartree-Fock model; $\mathrm{n}=$ neutrons; $\mathrm{p}=$ protons; $\mathrm{H}=$ hyperons, $\mathrm{K}=K^{-}[u, \bar{s}]$ meson condensate; $\mathrm{Q}=u, d, s$ quarks; H-matter=H-dibaryon condensate.

the emitted electromagnetic radiation, (2) accretion-powered (X-ray) pulsars, where the gravitational potential energy of the matter accreted from a low-mass companion is the energy source, and (3) magnetars (e.g, SGR 1806-20), where the decay of a ultra-strong magnetic field powers the radiation. The fastest, very recently discovered neutron star, PSR J1748-2446ad, rotates at a period of $1.39 \mathrm{~ms}$ (which corresponds to $719 \mathrm{~Hz}$ ] 9], followed by PSR B1937+21 10] and PSR B1957+20 [11] whose rotational periods are $1.58 \mathrm{~ms}(633 \mathrm{~Hz})$ and $1.61 \mathrm{~ms}(621 \mathrm{~Hz})$, respectively. PSR B1957+20 is moving through the galaxy at a speed of almost a million kilometers per hour. Due to this remarkable motion a bow shock wave is visible to optical telescopes. Other significant pulsars are Cen X-3 (first X-ray pulsar), SAX J1808.4-3658 (first accreting millisecond X-ray pulsar), and PSR J0737-3039A\&B (first double pulsar binary system, which will permit a strong-field test of General Relativity). When neutron stars are formed they have interior temperatures of the order of $10^{11} \mathrm{~K}$ (around $10 \mathrm{MeV}$ ). They cool by neutrino emission processes to interior temperatures of $\sim 10^{10} \mathrm{~K}$ within a few days. Throughout most of the active life of neutron stars in pulsars and X-ray sources, the interior temperature is $\sim 10^{7}-10^{9}$ K. Surface temperatures are an order of magnitude or more smaller. Measurements of the surface temperature of the Crab pulsar $(\mathrm{B} 0531+21)$, for instance, have led to $T<2 \times 10^{6} \mathrm{~K}$.) Rotating magnetized neutron stars in pulsars are surrounded by a plasma, the so-called magnetosphere, in which via plasma processes the electromagnetic radiation from pulsars is generated. The magnetic fields near the surface of neutron stars in compact X-ray sources play an important role in channeling the accreting matter onto the neutron star surface. To date over 1600 pulsars are known. This figure is expected to increase dramatically with the operation of instruments like the Square-Kilometer-Array (SkA). The sensitivity of SkA will be around 100 times higher than the VLA sensitivity; it is expected that around 20,000 new pulsars (including pulsars around black holes) will be discovered with SkA. The number of millisecond pulsars is expected to go up from its present value by a factor of 100 . The initial operations of SkA will start around 2016, and the final operations are expected to begin around 2020.

\section{Bounds on the Nuclear Equation of State}

In 1939, Tolman, Oppenheimer and Volkoff performed the first neutron star calculations, assuming that such objects are entirely made of a gas of non-interacting relativistic neutrons [12, 13]. The EoS of 


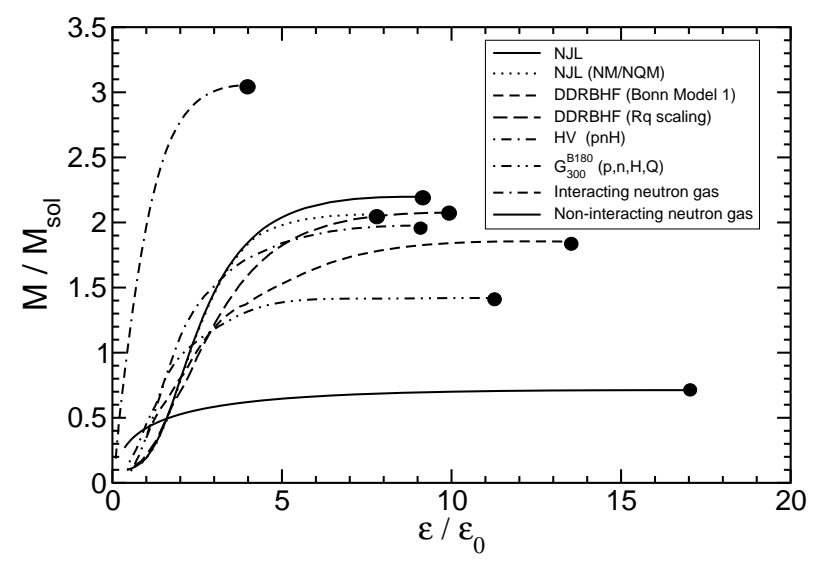

Figure 3: Neutron star mass versus central energy density $\left(\epsilon_{0}=140 \mathrm{MeV} / \mathrm{fm}^{3}\right)$.

such a gas is extremely soft, i.e. very little additional pressure is gained with increasing density, as can be seen from Fig. 2, and predicts a maximum neutron star mass of just $0.7 M_{\odot}$ (Figs. 3 and (4) at an unrealistically high density of 17 times the density of nuclear matter (Fig. 3). It is interesting to note that the inclusion of interactions among the neutrons increases the star's maximum mass from $0.7 M_{\odot}$ to around $3 M_{\odot}$ (Figs. 3 and 4 ). However, the radii of the latter stars are so big that mass shedding from the equator occurs for most stars of the sequence at rotational frequencies that are considerably smaller than $716 \mathrm{~Hz}$ observed for PSR J1748-2446ad, or B1937+21, $630 \mathrm{~Hz}$ (1.58 ms) [10]. An interacting neutron gas thus fails to accommodate the rotational frequencies of the most rapidly rotating, observed neutron stars. The other extreme, a non-interacting relativistic neutron gas, fails too since it does not accommodate the Hulse-Taylor pulsar $\left(M=1.44 M_{\odot}\right)$ [14], and also conflicts with the average neutron star mass of $1.350 \pm 0.004 M_{\odot}$ derived by Thorsett and Chakrabarty [15] from observations of radio pulsar systems. More than that, recent observations indicate that neutron star masses may be as high as around $2 M_{\odot}$. Examples of such very heavy neutron stars are $M_{\mathrm{J} 0751+1807}=2.1 \pm 0.2 M_{\odot}$ [16], $M_{4 \mathrm{U} 1636+536}=2.0 \pm 0.1 M_{\odot}$ [17], $M_{\text {VelaX }-1}=1.86 \pm 0.16 M_{\odot}$ [18], $M_{\text {Cyg X }-2}=1.78 \pm 0.23 M_{\odot}$ [19, 20]. Large masses have also been reported for the high-mass X-ray binary 4U 1700-37 and the compact object in the low-mass X-ray binary 2S0921-630, $M_{4 \mathrm{U} 1700-37}=2.44 \pm 0.27 M_{\odot}$ [21] and $M_{2 \mathrm{~S} 0921-630}=2.0-4.3 M_{\odot}[22$, respectively. The latter two objects may be either massive neutron stars or low-mass black holes with masses slightly higher than the maximum possible neutron star mass of $\sim 3 M_{\odot}$. This value follows from a general, theoretical estimate of the maximal possible mass of a stable neutron star [23]. If either one of the two objects $4 \mathrm{U} 1700-37$ or 2S0921-630 were a black hole, it would confirm the prediction of the existence of low-mass black holes [24]. Conversely, if these objects were massive neutron stars, their high masses would severely constrain the EoS of dense nuclear matter.

\section{Nuclear Many-Body Models}

A vast number of models for the equation of state (EoS) of neutron star matter has been derived in the literature over the years. The majority of these models belong to either one of the following categories: 1. Thomas-Fermi based models [25, 26] such as the new Thomas-Fermi approach of Myers and Swiatecki [25]. It is based on a Seyler-Blanchard potential generalized by the addition of one momentum dependent and one density dependent term

$$
V_{12}=-\frac{2 T_{0}}{\rho_{0}} Y\left(r_{12}\right)\left(\frac{1}{2}(1 \mp \xi) \alpha-\frac{1}{2}(1 \mp \zeta)\left(\beta\left(\frac{p_{12}}{k_{F_{0}}}\right)^{2}-\gamma \frac{k_{F_{0}}}{p_{12}}+\sigma\left(\frac{2 \bar{\rho}}{\rho_{0}}\right)^{\frac{2}{3}}\right)\right) .
$$


The upper (lower) sign in Eq. (11) corresponds to nucleons with equal (unequal) isospin. The quantities $k_{F_{0}}, T_{0}\left(=k_{F_{0}}^{2} / 2 m\right)$, and $\rho_{0}$ are the Fermi momentum, the Fermi energy and the saturation density of symmetric nuclear matter. The potential's radial dependence is described by a Yukawa-type interaction of the form $Y\left(r_{12}\right)=\left(4 \pi a^{3}\right)^{-1} \exp \left(-r_{12} / a\right) /\left(r_{12} / a\right)$. Its strength depends both on the magnitude of the particles' relative momentum, $p_{12}$, and on an average of the densities at the locations of the particles. The parameters $\xi$ and $\zeta$ were introduced in order to achieve better agreement with asymmetric nuclear systems. The behavior of the optical potential is improved by the term $\sigma\left(2 \bar{\rho} / \rho_{0}\right)^{2 / 3}$ with the average density defined as $\bar{\rho}^{2 / 3}=\left(\rho_{1}^{2 / 3}+\rho_{2}^{2 / 3}\right) / 2$, where $\rho_{1}$ and $\rho_{2}$ the densities of interacting neutron or protons at points 1 and 2 . The seven free parameters of the theory are adjusted to the properties of finite nuclei, the parameters of the mass formula, and the behavior of the optical potential [25].

2. Schroedinger-based models [3, 7, 27, 28, 29] are derived from Hamiltonians of the form

$$
\mathcal{H}=\sum_{i} \frac{-\hbar^{2}}{2 m} \nabla_{i}^{2}+\sum_{i<j} V_{i j}+\sum_{i<j<k} V_{i j k},
$$

where $V_{i j}$ and $V_{i j k}$ denote two and three-nucleon interactions. The many-body equations are then solved, for instance, in the framework of the hole-line expansion (Brueckner theory), coupled cluster method, self-consistent Green functions technique, or variational approach.

3. Relativistic nucler field-theoretical treatments such as relativistic mean field (RMF), Hartree-Fock (RHF), standard Brueckner-Hartree-Fock (RBHF), density dependent RBHF (DD-RBHF) [1, 2, 30, 31, 32, 33, 34, 35] which are based on a Lagrangian of the form $\mathcal{L}=\mathcal{L}_{B}+\mathcal{L}_{M}+\mathcal{L}_{\text {int }}+\mathcal{L}_{\text {lept }}$, where

$$
\begin{aligned}
\mathcal{L}_{B} & =\bar{\psi}\left(i \gamma_{\mu} \partial^{\mu}-m\right) \psi \\
\mathcal{L}_{M} & =\frac{1}{2} \sum_{i=\sigma, \delta}\left(\partial_{\mu} \Phi_{i} \partial^{\mu} \Phi_{i}-m_{i}^{2} \Phi_{i}^{2}\right)-\frac{1}{2} \sum_{\kappa=\omega, \rho}\left(\frac{1}{2} F_{\mu \nu}^{(\kappa)} F^{(\kappa) \mu \nu}-m_{\kappa}^{2} A_{\mu}^{(\kappa)} A^{(\kappa) \mu}\right), \\
\mathcal{L}_{i n t} & =\bar{\psi} \hat{\Gamma}_{\sigma}(\bar{\psi}, \psi) \psi \Phi_{\sigma}-\bar{\psi} \hat{\Gamma}_{\omega}(\bar{\psi}, \psi) \gamma_{\mu} \psi A^{(\omega) \mu}+\bar{\psi} \hat{\Gamma}_{\delta}(\bar{\psi}, \psi) \boldsymbol{\tau} \psi \boldsymbol{\Phi}_{\delta}-\bar{\psi} \hat{\Gamma}_{\rho}(\bar{\psi}, \psi) \gamma_{\mu} \boldsymbol{\tau} \psi \boldsymbol{A}^{(\rho) \mu} .
\end{aligned}
$$

Here, $\mathcal{L}_{B}$ and $\mathcal{L}_{M}$ are the free baryonic and the free mesonic Lagrangians, respectively, and interactions are described by $\mathcal{L}_{\text {int }}$, where $F_{\mu \nu}^{(\kappa)}=\partial_{\mu} A_{\nu}^{(\kappa)}-\partial_{\nu} A_{\mu}^{(\kappa)}$ is the field strength tensor of one of the vector mesons $(\kappa=\omega, \rho)$. In RMF, RHF and RBHF the meson-baryon vertices $\hat{\Gamma}_{\alpha}(\alpha=\sigma, \omega, \delta, \rho)$ are density-independent quantities which are given by expressions like $\hat{\Gamma}_{\sigma}=i g_{\sigma}$ for the scalar $\sigma$ meson, $\hat{\Gamma}_{\omega}^{\mu}=g_{\omega} \gamma^{\mu}+(i / 2)\left(f_{\omega} / 2 m\right) \partial_{\lambda}\left[\gamma^{\lambda}, \gamma^{\mu}\right]$ for $\omega$ mesons, etc. [2]. In the framework of the DD-RBHF scheme, the meson-baryon vertices $\hat{\Gamma}_{\alpha}$ are not only determined by Dirac matrices but depend on the baryon field operators $\psi[33]$.

4. The Nambu-Jona-Lasinio (NJL) model was originally introduced to describe nucleons with dynamically generated masses. In recent years it has become a popular model to describe quarks-hadron matter, and to explore the condensation patter of color superconducting quark matter [36, 37, 38, 39, 40, 41, 42, 43. The flavor SU(2) NJL-Lagrangian, for instance, which has been applied in [43] to derive both nuclear matter and quark matter phases, is given by

$$
\begin{aligned}
\mathcal{L}= & \bar{\psi}\left(i \not \partial-m_{q}\right) \psi+G_{\pi}\left((\bar{\psi} \psi)^{2}-\left(\bar{\psi} \gamma_{5} \boldsymbol{\tau} \psi\right)^{2}\right)-G_{\omega}\left(\bar{\psi} \gamma^{\mu} \psi\right)^{2}-G_{\rho}\left(\bar{\psi} \gamma^{\mu} \boldsymbol{\tau} \psi\right)^{2} \\
& +G_{s}\left(\bar{\psi} \gamma_{5} C \tau_{2} \beta^{A} \bar{\psi}^{T}\right)\left(\psi^{T} C^{-1} \gamma_{5} \tau_{2} \beta^{A} \psi\right) .
\end{aligned}
$$

Here $m_{q}$ is the current quark mass, $\psi$ is the flavor $\mathrm{SU}(2)$ quark field, and the coupling constants $G_{\pi}$, $G_{\omega}$ and $G_{\rho}$ characterize the $q \bar{q}$ interactions in the scalar, pseudoscalar and vector meson channels, while $G_{s}$ refers to the interaction in the scalar diquark channel [38, 43].

5. Aside from the NJL model, there are several other phenomenological models based on quark degrees of freedom, such as the quark meson coupling model, the cloudy bag model, the quark mean field model, and the chiral SU(3) quark mean field model [44. In the latter model, quarks are confined 


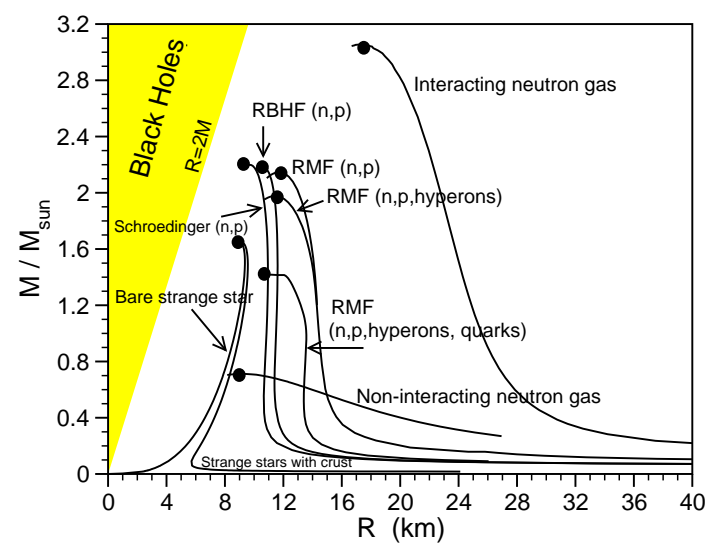

Figure 4: Mass-radius relationship of neutron stars and strange stars [6].

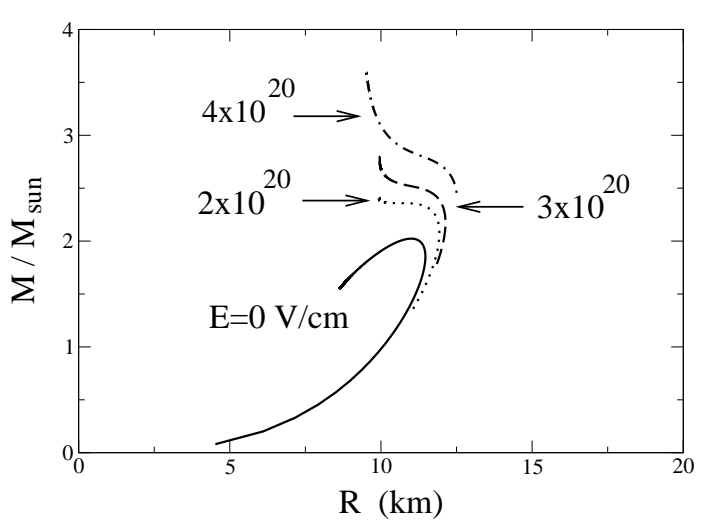

Figure 5: Mass-radius relationship of electrically charged strange stars.

within baryons by an effective potential. The quark-meson interaction and meson self-interaction are based on SU(3) chiral symmetry. The chiral SU(3) quark mean field model was applied recently to the study of neutron stars and strange stars [44].

A collection of equations of state computed for several of these models is shown in Fig. 2. Mass-radius relationships of neutron stars based on these EoS are shown in Figs. 4 and 5 . Strange star sequences are shown in these figures too. The strange star sequences shown in Fig. 5 show the impact of ultrastrong electric fields of the mass-radius relationship of strange stars. The energy-momentum tensor of such stars consists of the usual perfect-fluid term, which is supplemented with the electromagnetic energy-momentum tensor [45], $T_{\nu}{ }^{\mu}=\left(p+\rho c^{2}\right) u_{\nu} u^{\mu}+p \delta_{\nu}{ }^{\mu}+\left[F^{\mu l} F_{\nu l}+\delta_{\nu}{ }^{\mu} F_{k l} F^{k l} / 4 \pi\right] / 4 \pi$, where $u^{\mu}$ is the fluid's four-velocity, $p$ and $\rho c^{2} \equiv \epsilon$ are the pressure and energy density, respectively, and $F^{\mu \nu}$ satisfies the covariant Maxwell equation, $\left[(-g)^{1 / 2} F^{\nu \mu}\right]_{, \mu}=4 \pi J^{\nu}(-g)^{1 / 2}$. The total mass of the star, contained within a radial distance $r$ from the star's center, is given by $d m(r) / d r=\left(4 \pi \epsilon r^{2}\right) / c^{2}+\left(Q(r) / c^{2} r\right)(d Q(r) / d r)$. This relation shows that, in addition to the standard term originating from the EoS of the stellar fluid, the electric field energy too contributes to the star's total mass. The Tolman-Oppenheimer-Volkoff (TOV) equation of electrically charged stars is given by

$$
\frac{d p}{d r}=-\frac{2 G\left[m(r)+\frac{4 \pi r^{3}}{c^{2}}\left(p-\frac{Q^{2}(r)}{4 \pi r^{4} c^{2}}\right)\right]}{c^{2} r^{2}\left(1-\frac{2 G m(r)}{c^{2} r}+\frac{G Q^{2}(r)}{r^{2} c^{4}}\right)}(p+\epsilon)+\frac{Q(r)}{4 \pi r^{4}} \frac{d Q(r)}{d r} .
$$

As shown in [45], electric fields can substantially alter the structure of compact stars. This is specifically the case for the masses of neutron stars, provided they posses net electric charges. For strange stars, however, even the maximum possible electric fields, $\sim 10^{18} \mathrm{~V} / \mathrm{cm}$, modifies the mass-radius relationship only very weakly as can be seen from Fig. 囵,

\section{Building Blocks of Neutron Star Matter}

\subsection{Hyperons and baryon resonances}

At the densities in the interior of neutron stars, the neutron chemical potential, $\mu^{n}$, is likely to exceed the masses, modified by interactions, of $\Sigma, \Lambda$ and possibly $\Xi$ hyperons [2, 46]. Hence, in addition to nucleons, neutron star matter may be expected to contain significant populations of strangeness carrying hyperons. The thresholds of the lightest baryon resonances $\left(\Delta^{-}, \Delta^{0}, \Delta^{+}, \Delta^{++}\right)$are not reached in relativistic mean-field (RMF) calculations. This is different for many-body calculations performed 


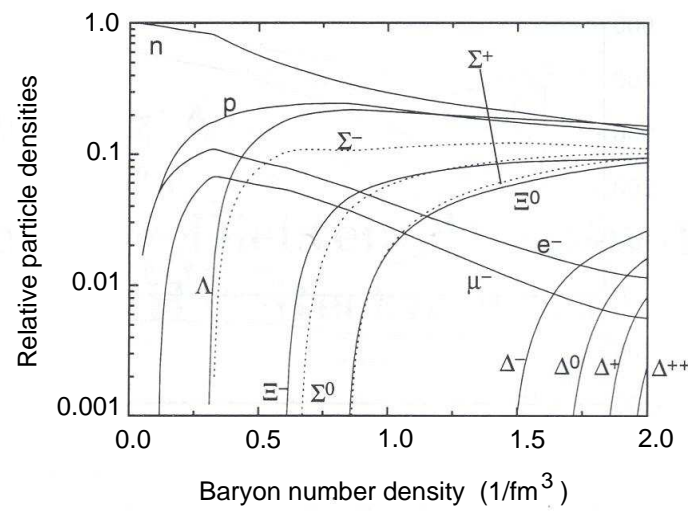

Figure 6: Composition of standard neutron star matter, i.e. $T=0 \mathrm{MeV}$ and $Y_{L}=0$ for the RMF approximation.

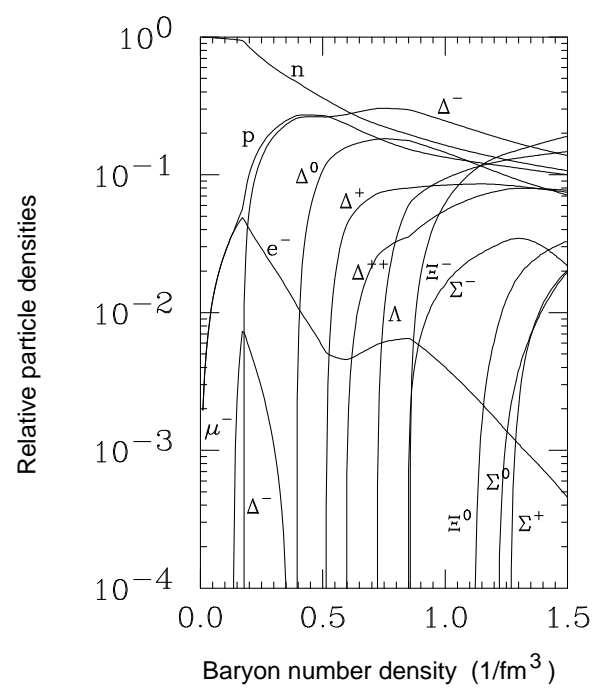

Figure 7: Same as Fig. 6, but computed for the RBHF approximation [47].

for the relativistic Brueckner-Hartree-Fock (RBHF) approximation where $\Delta$ 's appear rather abundantly [4], compare Figs. 6 and 7. Depending on the star's mass, the total hyperon population can be very large [46], which is illustrated graphically in Figs. 8 and 9 for rotating neutron stars whose EoS is computed in the framework of the relativistic DD-RBHF formalism [33]. The stars shown in these figures have rotational frequencies ranging from zero to the mass shedding frequency, $\nu_{\mathrm{K}}$, which is the maximum frequency a star can have before mass loss at the equator sets in. This frequency sets an absolute upper limit on stable rapid rotation and follows from a metric of the form [2, 48]

$$
d s^{2}=-e^{2 \nu} d t^{2}+e^{2 \psi}(d \phi-\omega d t)^{2}+e^{2 \mu} d \theta^{2}+e^{2 \lambda} d r^{2}
$$

where $\nu, \psi, \mu$ and $\lambda$ denote the metric functions which depend on the radial coordinate $r$, polar angle $\theta$ and, implicitly, on the star's angular velocity $\Omega$ as well as on the angular velocity $\omega$ at which the local inertial frames are dragged along in the direction of the star's rotation. In Newtonian mechanics, mass shedding is determined by the equality between centrifuge and gravity, and is readily obtained as $2 \pi \nu_{\mathrm{K}}=\sqrt{M / R^{3}}$. Its general relativistic counterpart, computed here, is given by [2, 48]

$$
2 \pi \nu_{\mathrm{K}}=\omega+\frac{\omega^{\prime}}{2 \psi^{\prime}}+e^{\nu-\psi}\left(\frac{\nu^{\prime}}{\psi^{\prime}}+\left(\frac{\omega^{\prime}}{2 \psi^{\prime}} e^{\psi-\nu}\right)^{2}\right)^{1 / 2}
$$

The primes denote derivatives with respect to the Schwarzschild radial coordinate. Finally, we take a brief look at the composition of proto-neutron star matter. The composition of such matter is determined by the requirements of charge neutrality and equilibrium under the weak processes, $B_{1} \rightarrow$ $B_{2}+l+\bar{\nu}_{l}$ and $B_{2}+l \rightarrow B_{1}+\nu_{l}$, where $B_{1}$ and $B_{2}$ are baryons, and $l$ is a lepton, either an electron or a muon. For standard neutron star matter, where the neutrinos have left the system, these two requirements imply that $Q=\sum_{i} q_{i} n_{B_{i}}+\sum_{l=e, \mu} q_{l} n_{l}=0$ (electric charge neutrality) and $\mu^{B_{i}}=b_{i} \mu^{n}-q_{i} \mu^{l}$ (chemical equilibrium), where $q_{i / l}$ denotes the electric charge density of a given particle, and $n_{B_{i}}\left(n_{l}\right)$ is the baryon (lepton) number density. The subscript $i$ runs over all the baryons considered. The symbol $\mu^{B_{i}}$ refers to the chemical potential of baryon $i, b_{i}$ is the particle's baryon number, and $q_{i}$ is its charge. The chemical potential of the neutron is denoted by $\mu^{n}$. When the neutrinos are trapped, as it is the case for proto-neutron star matter, the chemical equilibrium condition is altered to $\mu^{B_{i}}=b_{i} \mu^{n}-q_{i}\left(\mu^{l}-\mu^{\nu_{l}}\right)$ 


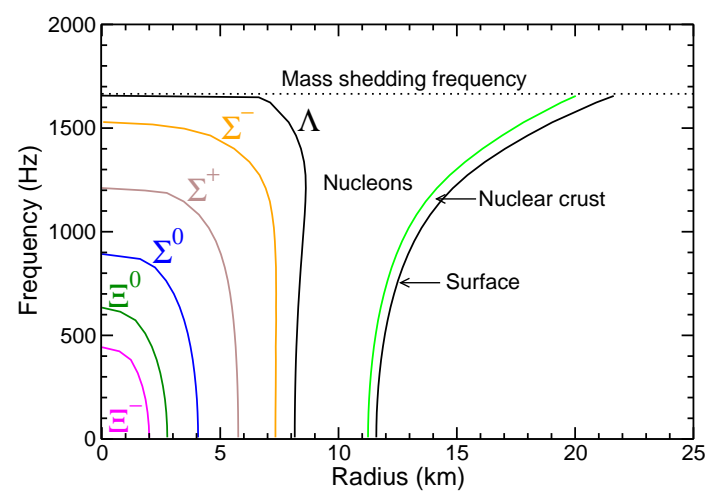

Figure 8: Hyperon composition of a rotating neutron star in equatorial direction.

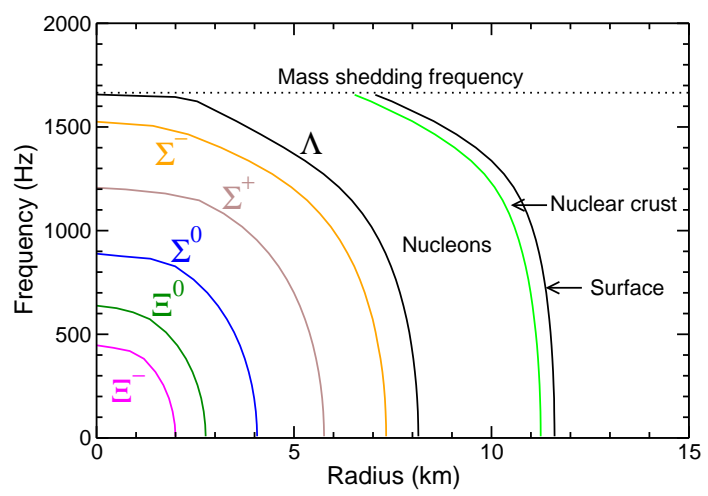

Figure 9: Same as Fig. 8, but in polar direction.

and $\mu^{e}-\mu^{\nu_{e}}=\mu^{\mu}-\mu^{\nu_{\mu}}$, where $\mu^{\nu_{l}}$ is the chemical potential of the neutrino $\nu_{l}$. In proto-neutron star matter, the electron lepton number $Y_{L}=\left(n_{e}+n_{\nu_{e}}\right) / n_{B}$ is initially fixed at a value of around $Y_{L_{e}}=Y_{e}+Y_{\nu_{e}} \simeq 0.3-0.4$ as suggested by gravitational collapse calculations of massive stars. Also, because no muons are present when neutrinos are trapped, the constraint $Y_{L_{\mu}}=Y_{\mu}+Y_{\nu_{m} u}=0$ can be imposed. Figures 10 and 11] show sample compositions of proto-neutron star matter and standard neutron star matter (no neutrinos) computed for the relativistic mean-field approximation. The presence of the $\Delta$ particle in (proto) neutron star matter at finite temperature is striking. As already mentioned at the beginning of this section, this particle is generally absent in cold neutron star matter treated in the relativistic mean-field approximation.

\subsection{Meson condensation}

The condensation of negatively charged mesons in neutron star matter is favored because such mesons would replace electrons with very high Fermi momenta. Early estimates predicted the onset of a negatively charged pion condensate at around $2 n_{0}$. However, these estimates are very sensitive to the strength of the effective nucleon particle-hole repulsion in the isospin $T=1$, spin $S=1$ channel, described by the Landau Fermi-liquid parameter $g^{\prime}$, which tends to suppress the condensation mechanism. Measurements in nuclei tend to indicate that the repulsion is too strong to permit condensation in nuclear matter [49, 50]. In the mid 1980s, it was discovered that the in-medium properties of $K^{-}[u \bar{s}]$ mesons may be such that this meson rather than the $\pi^{-}$meson may condense in neutron star matter [51, [52]. The condensation is initiated by the schematic reaction $e^{-} \rightarrow K^{-}+\nu_{e}$. If this reaction becomes possible in neutron star matter, it is energetically advantageous to replace the fermionic electrons with the bosonic $K^{-}$mesons. Whether or not this happens depends on the behavior of the $K^{-}$mass, $m_{K^{-}}^{*}$, in neutron star matter. Experiments which shed light on the properties of the $K^{-}$in nuclear matter have been performed with the Kaon Spectrometer (KaoS) and the FOPI detector at the heavy-ion synchrotron SIS at GSI [53]. An analysis of the early $K^{-}$kinetic energy spectra extracted from $\mathrm{Ni}+\mathrm{Ni}$ collisions showed that the attraction from nuclear matter would bring the $K^{-}$mass down to $m_{K^{-}}^{*} \simeq 200 \mathrm{MeV}$ at densities $\sim 3 n_{0}$. For neutron-rich matter, the relation $m_{K^{-}}^{*} / m_{K^{-}} \simeq 1-0.2 n / n_{0}$ was established [54], with $m_{K}=495 \mathrm{MeV}$ the $K^{-}$vacuum mass. Values of around $m_{K^{-}}^{*} \simeq 200 \mathrm{MeV}$ may be reached by the electron chemical potential, $\mu^{e}$, in neutron star matter [2, 46] so that the threshold condition for the onset of $K^{-}$condensation, $\mu^{e}=m_{K}^{*}$, might be fulfilled for sufficiently dense neutron stars, provided other negatively charged particles $\left(\Sigma^{-}, \Delta^{-}, d\right.$ and $s$ quarks) are not populated first and prevent the electron chemical potential from increasing with density. We also note that $K^{-}$condensation allows the conversion reaction $n \rightarrow p+K^{-}$. By this conversion the nucleons in the cores of neutron stars can 


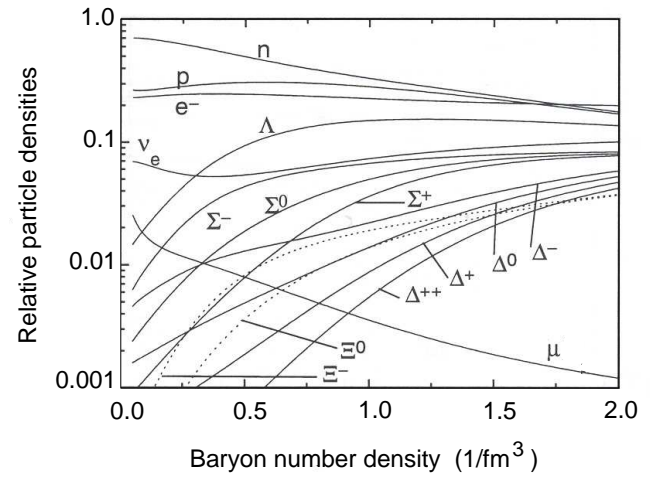

Figure 10: Composition of hot $(T=$ $40 \mathrm{MeV}$ ) proto-neutron star matter for $Y_{L}=0.3$ [57].

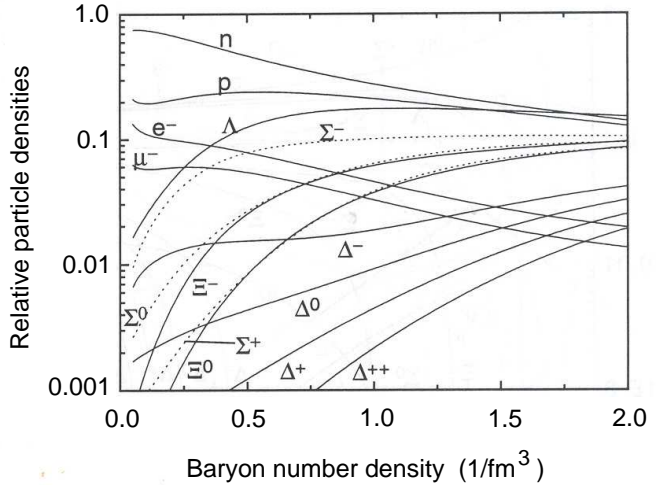

Figure 11: Same as Fig. 10, but for standard $\left(Y_{L}=0\right)$ neutron star matter [57].

become half neutrons and half protons, which lowers the energy per baryon of the matter. The relative isospin symmetric composition achieved in this way resembles the one of atomic nuclei, which are made up of roughly equal numbers of neutrons and protons. Neutron stars are therefore referred to, in this picture, as nucleon stars. The maximum mass of such stars has been calculated to be around $1.5 M_{\odot}$ [55. Consequently, the collapsing core of a supernova, e.g. 1987A, if heavier than this value, should go into a black hole rather than forming a neutron star, as pointed out by Brown et al. [24]. This would imply the existence of a large number of low-mass black holes in our galaxy [24]. Thielemann and Hashimoto [56] deduced from the total amount of ejected ${ }^{56} \mathrm{Ni}$ in supernova 1987A a neutron star mass range of $1.43-1.52 M_{\odot}$. If the maximum neutron star mass should indeed be in this mass range $\left(\sim 1.5 M_{\odot}\right)$, the existence of heavy neutron stars with masses around $2 M_{\odot}$ (Sect. 2) would be ruled out.

\subsection{H-matter and exotic baryons}

A novel particle that could be of relevance for the composition of neutron star matter is the H-dibaryon $(\mathrm{H}=([u d][d s][s u]))$, a doubly strange six-quark composite with spin and isospin zero, and baryon number two [58]. Since its first prediction in 1977, the H-dibaryon has been the subject of many theoretical and experimental studies as a possible candidate for a strongly bound exotic state. In neutron star matter, which may contain a significant fraction of $\Lambda$ hyperons, the $\Lambda$ 's could combine to form H-dibaryons, which could give way to the formation of H-dibaryon matter at densities somewhere above $\sim 4 n_{0}[59$. If formed in neutron stars, however, H-matter appears unstable against compression which could trigger the conversion of neutron stars into hypothetical strange stars [60, 61]. Another particle, referred to as an exotic baryon, of potential relevance for neutron stars, could be the pentaquark, $\Theta^{+}\left([u d]^{2} \bar{s}\right)$, with a predicted mass of $1540 \mathrm{MeV}$. The pentaquark, which carries baryon number one, is a hypothetical subatomic particle consisting of a group of four quarks and one anti-quark (compared to three quarks in normal baryons and two in mesons), bound by the strong color-spin correlation force (attraction between quarks in the color $\overline{\mathbf{3}}_{c}$ channel) that drives color superconductivity [62]. The pentaquark decays according to $\Theta^{+}(1540) \rightarrow K^{+}[\bar{s} u]+n[u d d]$ and thus has the same quantum numbers as the $K^{+} n$. 


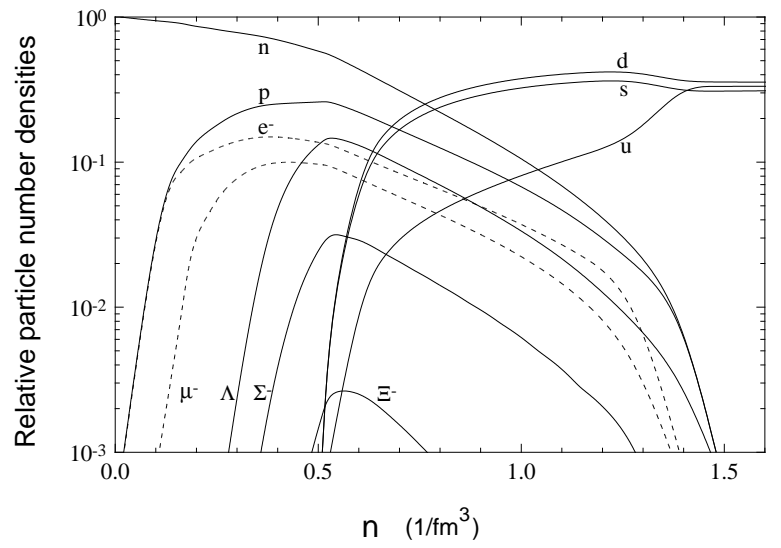

Figure 12: Sample composition of chemically equilibrated quark-hadron (hybrid star) matter as a function of baryon number density [2, 6].

\subsection{Quark deconfinement}

It has been suggested already several decades ago [63, 64, 65, 66, 67, 68, 69, 70] that the nucleons in the cores of neutron stars may melt under the enormous pressures that exist in the cores, creating a new state of matter know as quark matter. From simple geometrical considerations it follows that quark confinement could occur at densities somewhere between around $2-10 n_{0}$. Depending on rotational frequency and neutron star mass, densities greater than two to three times $n_{0}$ are easily reached in the cores of neutron stars so that the neutrons and protons in the cores of neutron stars may indeed be broken up into their quarks constituents [1, 2, 6, 71]. More than that, since the mass of the strange quark is only around $150 \mathrm{MeV}$, high-energetic up and down quarks will readily transform to strange quarks at about the same density at which up and down quark deconfinement sets in. Thus, if quark matter exists in the cores of neutron stars, it should be made of the three lightest quark flavors. The remaining three quark flavors (charm, top, bottom) are way to massive to be created in neutron stars. For instance, the creation of charm quark requires a density greater than $10^{17} \mathrm{~g} / \mathrm{cm}^{3}$, which around 100 times greater than the density reached in neutron stars. A stability analysis of stars with a charm quark population reveals that such objects are unstable against radial oscillations and, thus, can not exist stably in the Universe [2, 6]. The same is true for ultra-compact stars with unconfined populations of top and bottom quarks, since the pulsation eigen-equations are of Sturm-Liouville type.

The phase transition from confined hadronic $(\mathrm{H})$ matter to deconfined quark $(\mathrm{Q})$ matter is characterized by the conservation of baryon charge and electric charge. The Gibbs condition for phase equilibrium then is that the two associated chemical potentials, $\mu^{n}$ and $\mu^{e}$, and the pressure in the two phases be equal [71], $P_{\mathrm{H}}\left(\mu^{n}, \mu^{e},\left\{\psi_{\chi}\right\}, T\right)=P_{\mathrm{Q}}\left(\mu^{n}, \mu^{e}, T\right)$, where $P_{\mathrm{H}}$ denotes the pressure of hadronic matter computed for a given hadronic Lagrangian $\mathcal{L}_{\mathrm{M}}\left(\left\{\psi_{\chi}\right\}\right)$, with $\left\{\psi_{\chi}\right\}$ the field variables and Fermi momenta that characterize a solution to the field equations of confined hadronic matter,

$$
\begin{aligned}
\left(i \gamma^{\mu} \partial_{\mu}-m_{\chi}\right) \psi_{\chi}(x) & =\sum_{M=\sigma, \omega, \pi, \ldots} \hat{\Gamma}_{M \chi} M(x) \psi_{\chi}(x) \\
\left(\partial^{\mu} \partial_{\mu}+m_{\sigma}^{2}\right) \sigma(x) & =\sum_{\chi=p, n, \Sigma, \ldots} \hat{\Gamma}_{\sigma \chi} \bar{\psi}_{\chi}(x) \psi_{\chi}(x), \quad \text { etc. }
\end{aligned}
$$

The pressure of quark matter, $P_{\mathrm{Q}}$, is obtainable from the bag model. The quark chemical potentials $\mu^{u}, \mu^{d}, \mu^{s}$ are related to the baryon and charge chemical potentials as $\mu^{u}=\left(\mu^{n}-2 \mu^{e}\right) / 3$ and $\mu^{d}=$ $\mu^{s}=\left(\mu^{n}+\mu^{e}\right) / 3$. The Gibbs condition is to be supplemented with the global relations for conservation of baryon charge and electric charge within an unknown volume $V$ containing $A$ baryons. The first 

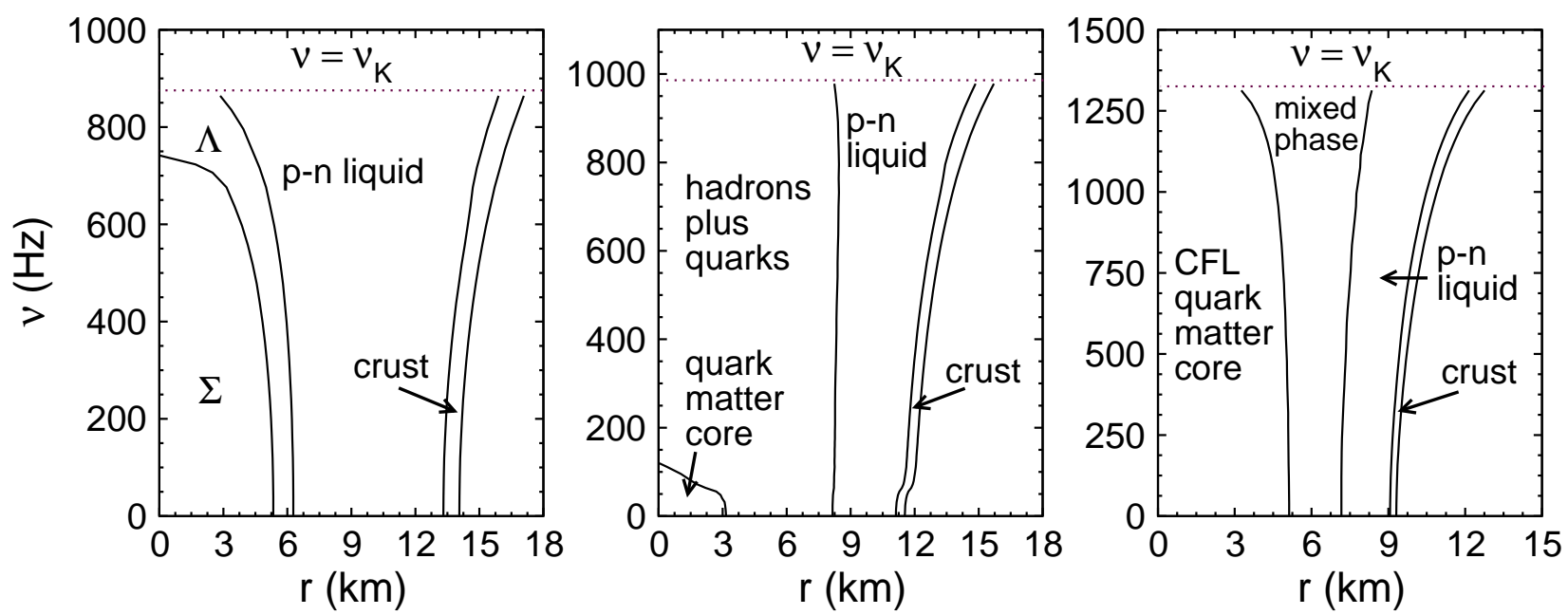

Figure 13: Dependence of neutron star composition on spin frequency, $\nu$, for three sample compositions (left: hyperon composition, middle: quark-hybrid composition, right: quarkhybrid composition with quark matter in the color-flavor locked phase). The non-rotating stellar mass in each case is $1.4 M_{\odot} . \nu_{\mathrm{K}}$ denotes the Kepler (mass-shedding) frequency.

one is given by $n \equiv A / V=(1-\chi) n_{\mathrm{H}}\left(\mu^{n}, \mu^{e}, T\right)+\chi n_{\mathrm{Q}}\left(\mu^{n}, \mu^{e}, T\right)$, where $\chi \equiv V_{\mathrm{Q}} / V$ denotes the volume proportion of quark matter, $V_{\mathrm{Q}}$, in the unknown volume $V$, and $n_{\mathrm{H}}$ and $n_{\mathrm{Q}}$ are the baryon number densities of hadronic matter and quark matter. Global neutrality of electric charge within the volume $V$ can be written as $0=Q / V=(1-\chi) q_{\mathrm{H}}\left(\mu^{n}, \mu^{e}, T\right)+\chi q_{\mathrm{Q}}\left(\mu^{n}, \mu^{e}, T\right)+q_{\mathrm{L}}$, with $q_{i}$ the electric charge densities of hadrons, quarks, and leptons. For a given temperature, $T$, these equations serve to determine the two independent chemical potentials and the volume $V$ for a specified volume fraction $\chi$ of the quark phase in equilibrium with the hadronic phase. After completion $V_{\mathrm{Q}}$ is obtained as $V_{\mathrm{Q}}=\chi V$. The chemical potentials depend on the proportion $\chi$ of the phases in equilibrium, and hence so do all properties that depend on them, i.e. the energy densities, baryon and charge densities of each phase, and the common pressure. For the mixed phase, the volume proportion of quark matter varies from $0 \leq \chi \leq 1$ and the energy density is the linear combination of the two phases $\epsilon=(1-\chi) \epsilon_{\mathrm{H}}\left(\mu^{n}, \mu^{e},\left\{\psi_{\chi}\right\}, T\right)+\chi \epsilon_{\mathrm{Q}}\left(\mu^{n}, \mu^{e}, T\right)$. Model neutron star compositions computed within the framework described just above are shown in Figs. 12 and 13. Possible astrophysical signals associated with quark deconfinement, the most striking of which being "backbending" of isolated pulsars, are discussed in [1, 2, 6, 72].

\subsection{Color-superconductivity of quark matter}

There has been much recent progress in our understanding of quark matter, culminating in the discovery that if quark matter exists it ought to be in a color superconducting state [36, 73, 74, 75]. This is made possible by the strong interaction among the quarks which is very attractive in some channels (antisymmetric antitriplet channel). Pairs of quarks are thus expected to form Cooper pairs very readily. Since pairs of quarks cannot be color-neutral, the resulting condensate will break the local color symmetry and form what is called a color superconductor. The phase diagram of such matter is expected to be very complex [73, 74]. The complexity is caused by the fact that quarks come in three different colors, different flavors, and different masses. Moreover, bulk matter is neutral with respect to both electric and color charge, and is in chemical equilibrium under the weak interaction processes that turn one quark flavor into another. To illustrate the condensation pattern briefly, we note the following pairing ansatz for the quark condensate $\left\langle\psi_{f_{a}}^{\alpha} C \gamma_{5} \psi_{f_{b}}^{\beta}\right\rangle \sim \Delta_{1} \epsilon^{\alpha \beta 1} \epsilon_{f_{a} f_{b} 1}+\Delta_{2} \epsilon^{\alpha \beta 2} \epsilon_{f_{a} f_{b} 2}+\Delta_{3} \epsilon^{\alpha \beta 3} \epsilon_{f_{a} f_{b} 3}$, where 
$\psi_{f_{a}}^{\alpha}$ is a quark of color $\alpha=(r, g, b)$ and flavor $f_{a}=(u, d, s)$. The condensate is a Lorentz scalar, antisymmetric in Dirac indices, antisymmetric in color, and thus antisymmetric in flavor. The gap parameters $\Delta_{1}, \Delta_{2}$ and $\Delta_{3}$ describe $d-s, u$-s and $u$ - $d$ quark Cooper pairs, respectively. The following pairing schemes have emerged: At asymptotic densities $\left(m_{s} \rightarrow 0\right.$ or $\left.\mu \rightarrow \infty\right)$ the ground state of QCD with a vanishing strange quark mass is the color-flavor locked (CFL) phase (color-flavor locked quark pairing), in which all three quark flavors participate symmetrically. The gaps associated with this phase are $\Delta_{3} \simeq \Delta_{2}=\Delta_{1}=\Delta$, and the quark condensates of the CFL phase are approximately of the form $\left\langle\psi_{f_{a}}^{\alpha} C \gamma_{5} \psi_{f_{b}}^{\beta}\right\rangle \sim \Delta \epsilon^{\alpha \beta X} \epsilon_{f_{a} f_{b} X}$, with color and flavor indices all running from 1 to 3 . Since $\epsilon^{\alpha \beta X} \epsilon_{f_{a} f_{b} X}=\delta_{f_{a}}^{\alpha} \delta_{f_{b}}^{\beta}-\delta_{f_{b}}^{\alpha} \delta_{f_{a}}^{\beta}$ one sees that the condensate involves Kronecker delta functions that link color and flavor indices. Hence the notion color-flavor locking. The CFL phase has been shown to be electrically neutral without any need for electrons for a significant range of chemical potentials and strange quark masses [76]. If the strange quark mass is heavy enough to be ignored, then up and down quarks may pair in the two-flavor superconducting (2SC) phase. Other possible condensation patterns are CFL- $K^{0}, \mathrm{CFL}-K^{+}$and CFL- $\pi^{0,-}$, gCFL (gapless CFL phase), 1SC (single-flavor-pairing), CSL (color-spin locked phase), and the LOFF (crystalline pairing) phase, depending on $m_{s}, \mu$, and electric charge density (for references, see [6]). Calculations performed for massless up and down quarks and a very heavy strange quark mass $\left(m_{s} \rightarrow \infty\right)$ agree that the quarks prefer to pair in the two-flavor superconducting (2SC) phase where $\Delta_{3}>0$, and $\Delta_{2}=\Delta_{1}=0$. In this case the pairing ansatz reduces to $\left\langle\psi_{f_{a}}^{\alpha} C \gamma_{5} \psi_{f_{b}}^{\beta}\right\rangle \propto \Delta \epsilon_{a b} \epsilon^{\alpha \beta 3}$. Here the resulting condensate picks a color direction (3 or blue in the example above), and creates a gap $\Delta$ at the Fermi surfaces of quarks with the other two out of three colors (red and green). The gapless CFL phase (gCFL) may prevail over the CFL and 2SC phases at intermediate values of $m_{s}^{2} / \mu$ with gaps given obeying the relation $\Delta_{3}>\Delta_{2}>\Delta_{1}>0$. For chemical potentials that are of astrophysical interest, $\mu<1000 \mathrm{MeV}$, the gap is between 50 and $100 \mathrm{MeV}$. The order of magnitude of this result agrees with calculations based on phenomenological effective interactions [75, 77] as well as with perturbative calculations for $\mu>10 \mathrm{GeV}$ [78]. We also note that superconductivity modifies the EoS at the order of $(\Delta / \mu)^{2}$ [79, 80], which is even for such large gaps only a few percent of the bulk energy. Such small effects may be safely neglected in present determinations of models for the EoS of quark-hybrid stars. There has been much recent work on how color superconductivity in neutron stars could affect their properties [73, 74, 81, 82, 83, 84]. These studies reveal that possible signatures include the cooling by neutrino emission, the pattern of the arrival times of supernova neutrinos, the evolution of neutron star magnetic fields, rotational stellar instabilities, and glitches in rotation frequencies.

\subsection{Absolutely stable strange quark matter}

It is most intriguing that for strange quark matter made of more than a few hundred up, down, and strange quarks, the energy of strange quark matter may be well below the energy of nuclear matter, $E / A=930 \mathrm{MeV}$, which gives rise to new and novel classes of strange matter objects, ranging from strangelets at the low baryon-number end to strange stars at the high baryon number end [2, 6, 85, 86, 87]. The presence of electrons in strange quark matter may lead to the formation of an electric dipole layer on the surface of strange matter, with huge electric fields on the order of $10^{19} \mathrm{~V} / \mathrm{cm}$. This peculiar feature enables strange quark stars to be enveloped in nuclear crusts made of ordinary atomic nuclei [85, 88, 89]. Sequences of compact strange stars with and without (bare) nuclear crusts are shown in Fig. 4. Since the nuclear crust is gravitationally bound to the quark matter, the mass-radius relationship of strange stars with crusts resembles the one of neutron stars and even that of white dwarfs [90, 91]. In contrast to neutron stars, however, strange stars obey $M \propto R^{3}$ because they are self-bound and the mass density of quark matter is almost constant inside strange stars. The electrons surrounding strange quark matter are held to quark matter electrostatically. Since neither component, electrons nor quark matter, is held in place gravitationally, the Eddington limit to the luminosity that 
a static surface may emit does not apply, and thus the object may have photon luminosities much greater than $10^{38} \mathrm{erg} / \mathrm{s}$. It was shown by Usov [92 that this value may be exceeded by many orders of magnitude by the luminosity of $e^{+} e^{-}$pairs produced by the Coulomb barrier at the surface of a hot strange star. For a surface temperature of $\sim 10^{11} \mathrm{~K}$, the luminosity in the outflowing pair plasma was calculated to be as high as $\sim 3 \times 10^{51} \mathrm{erg} / \mathrm{s}$. Such an effect may be a good observational signature of bare strange stars [92, 93, 94, 95. If the strange star is enveloped by a nuclear crust however, which is gravitationally bound to the strange star, the surface made up of ordinary atomic matter would be subject to the Eddington limit. Hence the photon emissivity of such a strange star would be the same as for an ordinary neutron star. If quark matter at the stellar surface is in the CFL phase the process of $e^{+} e^{-}$pair creation at the stellar quark matter surface may be turned off, since cold CFL quark matter is electrically neutral so that no electrons are required and none are admitted inside CFL quark matter [76]. This may be different for the early stages of a hot CFL quark star [96].

\section{$5 \quad$ Latent Heat of Phase Transitions}

A neutron star is born with enormous reserves of rotational and thermal energy, which it looses over millions of years through processes such as magnetic dipole radiation from the magnetosphere, thermal radiation and electron winds from the surface and neutrinos from the core. In doing so the star cools down and changes structure as the radius diminishes with the rotation frequency-by as much as a few kilometers if it started out near its limiting Kepler frequency-shifting the interior boundaries of any phase transitions there in the process. Any latent heat evolved or absorbed by particles crossing these boundaries will contribute to the thermal evolution af the star, and in this section we discuss the formalism for describing these effects. The thermal evolution of a spherical star in General Relativity is given by a local energy balance equation [97, 98]:

$$
\frac{e^{-\lambda-2 \phi}}{4 \pi r^{2}} \frac{\partial\left(L_{r} e^{2 \phi}\right)}{\partial r}=\epsilon_{\mathrm{nuc}}-\epsilon_{\nu}-\rho e^{-\phi} \frac{\partial \Pi}{\partial t}+e^{-\phi} \frac{P}{\rho} \frac{\partial \rho}{\partial t}, \quad L_{r}=4 \pi \kappa r^{2} e^{-\lambda-\phi} \frac{\partial\left(T e^{\phi}\right)}{\partial r},
$$

where $L_{r}$ is the local luminosity given by the conduction of non-neutrino energy through a sphere of radius $r, \epsilon_{n u c}$ is the rate, per volume, at which nuclear reactions create non-nuclear energy, $\epsilon_{\nu}$ is the neutrino emissivity per volume, $\rho$ is the rest mass density, $P$ is the pressure and $\Pi$ is the specific internal energy per unit rest mass, which in the absence of any phase transitions may be written

$$
\Pi=\frac{1}{\rho} \int_{0}^{T} c_{\mathrm{V}}\left(\rho, T^{\prime}\right) \mathrm{d} T^{\prime}
$$

with $c_{\mathrm{V}}$ the heat capacity per unit volume and $T$ the temperature. Since $P=-(\partial E / \partial V)_{T}$ we then have $\partial \Pi / \partial \rho=P / \rho^{2}$ so in this case

$$
\frac{\partial \Pi}{\partial t}=\frac{\partial \Pi}{\partial T} \frac{\partial T}{\partial t}+\frac{\partial \Pi}{\partial \rho} \frac{\partial \rho}{\partial t}=\frac{c_{\mathrm{V}}}{\rho} \frac{\partial T}{\partial t}+\frac{P}{\rho^{2}} \frac{\partial \rho}{\partial t},
$$

which gives the usual heat balance equation for neutron stars

$$
\frac{e^{-\lambda-2 \phi}}{4 \pi r^{2}} \frac{\partial\left(L_{r} e^{2 \phi}\right)}{\partial r}=\epsilon_{\mathrm{nuc}}-\epsilon_{\nu}-e^{-\phi} c_{\mathrm{V}} \frac{\partial T}{\partial t}
$$

However in the presence of any phase transitions the heat capacity may be discontinuous and this will give rise to extra terms as particles cross the phase boundaries in either temperature for superfluid

transitions or in density if there is a transition from hadronic matter to quark matter. To see this for a sharp phase transition with no mixed phase, we may write $\Pi$ as

$$
\Pi=\rho^{-1}\left[\Theta\left(1-\lambda / \lambda_{c, 1}\right) \int_{0}^{T} c_{\mathrm{V}, 1} \mathrm{~d} T^{\prime}+\Theta\left(\lambda / \lambda_{c, 2}-1\right) \int_{0}^{T} c_{\mathrm{V}, 2} \mathrm{~d} T^{\prime}\right],
$$


where $\Theta$ is the Heaviside step function, $\lambda$ can be either $T, \rho$ (which may be discontinous at the phase transition) or some other convenient quantity depending on the nature of the phase transition, $\lambda_{c}$ is the critical $\lambda$ for the phase transition and numerical subscripts identifies a variable with either of the two phases. Differentiating with respect to time we then get the same terms as above and additional terms from the Heaviside functions:

$$
\frac{\partial \Pi}{\partial t}=\frac{c_{\mathrm{V}}}{\rho} \frac{\partial T}{\partial t}+\frac{P}{\rho^{2}} \frac{\partial \rho}{\partial t}+\frac{1}{\rho}\left[\frac{\delta\left(\lambda / \lambda_{c, 2}-1\right)}{\lambda_{c, 2}} \frac{\partial \lambda}{\partial t} \int_{0}^{T} c_{\mathrm{V}, 2} \mathrm{~d} T^{\prime}-\frac{\delta\left(\lambda / \lambda_{c, 1}-1\right)}{\lambda_{c, 1}} \frac{\partial \lambda}{\partial t} \int_{0}^{T} c_{\mathrm{V}, 1} \mathrm{~d} T^{\prime}\right] .
$$

Simplifying this we note that $c_{\mathrm{V}}=T(\partial s / \partial T)_{\mathrm{V}}$ where $s$ is the entropy density, integrate by parts and use the phase equilibrium conditions that $P_{1}=P_{2}, T_{1}=T_{2}, \mu_{1}=\mu_{2}$ to get

$$
\frac{\partial \Pi}{\partial t}=\frac{c_{\mathrm{V}}}{\rho} \frac{\partial T}{\partial t}+\frac{P}{\rho^{2}} \frac{\partial \rho}{\partial t}+\frac{1}{\rho} \frac{\partial \lambda}{\partial t} T\left[\frac{\delta\left(\lambda / \lambda_{c, 2}-1\right)}{\lambda_{c, 2}} s_{2}-\frac{\delta\left(\lambda / \lambda_{c, 1}-1\right)}{\lambda_{c, 1}} s_{1}\right] .
$$

This is of course the well known result that each particle crossing a phase boundary contributes to the surrounding medium an energy [99] $q=-T\left[\sigma_{2}-\sigma_{1}\right]=-T \Delta \sigma$, where $\sigma$ is the entropy per particle. If $\lambda$ is a continuous variable such as $T$ or $P$ the distinction between $\lambda_{c, 1}$ and $\lambda_{c, 2}$ is pointless and the delta functions may be taken outside, and a second order transition with continuous entropy density at the phase transition would then cause the last term in Eq. (18) to vanish.

A mixed phase may be treated similarly by replacing the Heaviside functions with a volume fraction $\chi$, such that $1-\chi$ is the fraction of a given volume occupied by phase 1 and $\chi$ is that occupied by phase 2 so $\Pi=(1-\chi) \Pi_{1}+\chi \Pi_{2}$ and hence

$$
\frac{\partial \Pi}{\partial t}=\frac{c_{\mathrm{V}}}{\rho} \frac{\partial T}{\partial t}+\frac{P}{\rho^{2}} \frac{\partial \rho}{\partial t}+\frac{1}{\rho} \frac{\partial \chi}{\partial \lambda} \frac{\partial \lambda}{\partial t} T\left[s_{2}-s_{1}\right] .
$$

The transition to a superfluid state is a second order transition taking place through a mixed phase with $\Delta \sigma \propto-\left(1-T / T_{c}\right)$ and $\lim _{T \rightarrow 0} \chi=1-\left(2 \pi \Delta_{0} / T\right)^{1 / 2} e^{-\Delta_{0} / T}$ for simple Fermi gasses [99]. The latent heat released in this process is customarily treated as an increase in the heat capacity letting $c_{\mathrm{V}} \rightarrow c_{\mathrm{V}} R\left(T / T_{c}\right)$ with the effect of slightly slowing the cooling process - at temperatures around $0.2 T_{c}$ the reduction factor, $R\left(T / T_{c}\right)$, goes to zero and has the opposite effect of significantly accelerating the cooling. This has been extensively treated in the literature and we refer to [100] for a recent review.

The transition from hadronic to quark matter is a first order transition which will take place through a mixed phase [71] if finite size effects and charge screening do not prohibit such a phase [101, 102] (although see the comments in [103]). If the transition is sharp it happens at a specific critical pressure, where the density will be discontinuous and heat will be released there at a total rate of $-e^{-\phi} T \Delta \sigma\left(\mathrm{d} N_{\mathrm{q}} / \mathrm{d} t\right)$, with $\mathrm{d} N_{\mathrm{q}} / \mathrm{d} t$ the number of particles making the transition per unit time. If the transition is through a mixed phase the pressure and average entropy density, $s=(1-\chi) s_{1}+\chi s_{2}$, vary continously in the region where the phase transition takes place, but the individual entropy densities will not be equal and particles making the transition will release heat at a local rate of $-e^{-\phi}(\partial \chi / \partial t) T \Delta s$, where $\chi$ will increase as the star becomes more dense.

To estimate whether the contribution from these terms makes any significant difference or we are just carrying coal to Newcastle, we note that a transition from hadronic to quark matter may in a rough approximation be seen as a transition between free Fermi gasses at different densities-one of them with a bag constant in the energy density, but this does not affect the entropy density. Assuming both gasses are relativistic and degenerate the entropy per particle with Boltzmann's constant set to one is [99]

$$
\sigma=\gamma_{c} \frac{\left(3 \pi^{2}\right)^{\frac{2}{3}}}{3 \hbar c} T n^{-\frac{1}{3}} \simeq \gamma_{c} 0.02 \frac{T}{\mathrm{MeV}}\left(\frac{n}{\mathrm{fm}^{-3}}\right)^{-\frac{1}{3}}
$$


where $\gamma_{c}$ is the color degeneracy, $n$ is the particle density which for quark matter is three times the baryon density, $n_{\mathrm{QM}}$, in the quark phase and in the hadronic phase is equal to the baryon density there, $n_{\mathrm{H}}$. The resulting term in in Eq. (12) is then on the order of

$$
10^{33} \frac{T_{9}^{2}}{e^{\phi}}\left[\left(\frac{n_{\mathrm{H}}}{\mathrm{fm}^{-3}}\right)^{-\frac{1}{3}}-\left(\frac{3 n_{\mathrm{QM}}}{\mathrm{fm}^{-3}}\right)^{-\frac{1}{3}}\right] \frac{\mathrm{d} N_{q} / \mathrm{d} t}{10^{57} / 10^{7} \mathrm{yr}} \mathrm{erg} \mathrm{s}^{-1},
$$

where $T_{9}=T / 10^{9} \mathrm{~K}$ and $\mathrm{d} N_{q} / \mathrm{d} t$ is normalized to a whole star converted on a timescale of $10^{7}$ years. For the quarks $\gamma_{c}=3$ was canceled by a factor $1 / 3$ since it takes 3 quarks to make a baryon. This is a rather small contribution unless the temperature is high or the star is changing structure fast. Furthermore the term is only positive if $n_{\mathrm{H}}<3 n_{\mathrm{QM}}$, but this condition is of course subject to the specific assumptions we made here and would change in a more detailed treatment of the EoS. It should also be noted that this treatment accounts only for the latent heat from the deconfinement of the quarks, and that there would be further contributions from the subsequent weak reactions required to bring the matter into chemical equilibrium by converting $d$-quarks to $s$-quarks. These reactions would contribute to the $\epsilon_{\text {nuc }}$ and $\epsilon_{\nu}$ terms in Eq. (12) and must be treated similarly to the rotochemical heating discussed by [104]. As shown by [105] it is furthermore very important to know exactly where in the star a heating source is located, since sources located below the outer crust of the star tend to contribute only to the neutrino luminosity and make little difference to the surface temperature. In spite of such reservations one might still optimistically expect to find signals from the latent heat of a quark matter phase transition either early in a star's thermal history, when it is hot and spinning down rapidly, or from later episodes of rapid structure change. Such rapid structure change could result from glitches, which may be associated with the buildup and release of stress in a crystalline mixed quark hadron phase [106], or the appearance of quark matter in a neutron star core, which softens the equations of state and allows the star to change structure rapidly possibly even through a core quake if a metastable state can be accessed [107]. Future work will explore these possibilities.

\section{Summary}

Obviously, our view of the interior composition of pulsars, which contain matter in one of the densest forms found in the Universe, has changed dramatically since their first discovery some 40 years ago. It has also become clear during that time period that all the ambient conditions that characterize pulsars tend to the extreme as well, rendering pulsars to almost ideal astrophysical laboratories for a broad range of physical studies. Owing to the unprecedented wealth of high-quality data on pulsars provided by radio telescopes, X-ray satellites-and soon the latest generation of gravitational-wave detectors-it seems within reach to decipher the inner workings of these enigmatic objects, and to explore the phase diagram of cold and ultra-dense hadronic matter from astrophysics.

\section{Acknowledgments}

The research of F. Weber is supported by the National Science Foundation under Grant PHY-0457329, and by the Research Corporation.

\section{References}

[1] N. K. Glendenning, Compact Stars, Nuclear Physics, Particle Physics, and General Relativity, 2nd ed. (Springer-Verlag, New York, 2000). 
[2] F. Weber, Pulsars as Astrophysical Laboratories for Nuclear and Particle Physics, High Energy Physics, Cosmology and Gravitation Series (IOP Publishing, Bristol, Great Britain, 1999).

[3] H. Heiselberg and V. Pandharipande, Ann. Rev. Nucl. Part. Sci. 50 (2000) 481.

[4] J. M. Lattimer and M. Prakash, Astrophys. J. 550 (2001) 426.

[5] Physics of Neutron Star Interiors, ed. by D. Blaschke, N. K. Glendenning, and A. Sedrakian, Lecture Notes in Physics 578 (Spring-Verlag, Berlin, 2001).

[6] F. Weber, Prog. Part. Nucl. Phys. 54 (2005) 193, astro-ph/0407155).

[7] A. Sedrakian, The physics of dense hadronic matter and compact stars, (nucl-th/0601086).

[8] T. Klahn et al., Phys. Rev. C 74 (2006) 035802.

[9] J. W. T. Hessels, S. M. Ransom, I. H. Stairs, P. C. C. Freire, V. M. Kaspi, and F. Camilo, Science 311 (2006) 1901.

[10] D. C. Backer, S. R. Kulkarni, C. Heiles, M. M. Davis, and W. M. Goss, Nature 300 (1982) 615.

[11] A. S. Fruchter, D. R. Stinebring, and J. H. Taylor, Nature 334 (1988) 237.

[12] J. R. Oppenheimer and G. M. Volkoff, Phys. Rev. 55 (1939) 374.

[13] R. C. Tolman, Phys. Rev. 55 (1939) 364.

[14] J. H. Taylor and J. M. Weisberg, Astrophys. J. 345 (1989) 434.

[15] S. E. Thorsett and D. Chakrabarty, Astrophys. J. 512 (1999) 288.

[16] D. J. Nice, E. M. Splaver, I. H. Stairs, O. Loehmer, A. Jessner, M. Kramer, and J. M. Cordes, A 2.1 solar mass pulsar measured by relativistic orbital decay, (astro-ph/0508050).

[17] D. Barret, J.-F. Olive, and M. C. Miller, The coherence of kHz quasi-periodic oscillations in the $X$-rays from accreting neutron stars, (astro-ph/0605486).

[18] O. Barziv, L. Kaper, M. H. van Kerkwijk, J. H. Telting, and J. van Paradijs, Astron. \& Astrophys. 377 (2001) 925.

[19] J. Casares, P. A. Charles, and E. Kuulkers, Astrophys. J. 493 (1998) L39.

[20] J. A. Orosz and E. Kuulkers, Mon. Not. R. Astron. Soc. 305 (1999) 132.

[21] J. S. Clark, S. P. Goodwin, P. A. Crowther, L. Kaper, M. Fairbairn, N. Langer, and C. Brocksopp, Astron. \& Astrophys. 392 (2002) 909.

[22] T. Shahbaz, J. Casares, C. A. Watson, P. A. Charles, R. I. Hynes, S. C. Shih, and D. Steeghs, Astrophys. J. 616 (2004) L123.

[23] C. E. Rhoades and R. Ruffini, Phys. Rev. Lett. 32 (1974) 324.

[24] G. E. Brown and H. A. Bethe, Astrophys. J. 423 (1994) 659.

[25] W. D. Myers and W. J. Swiatecki, Nucl. Phys. A601 (1996) 141.

[26] K. Strobel, F. Weber, M. K. Weigel, and Ch. Schaab, Int. J. Mod. Phys. E 6, No. 4 (1997) 669. 
[27] V. R. Pandharipande and R. B. Wiringa, Rev. Mod. Phys. 51 (1979) 821.

[28] R. B. Wiringa, V. Fiks, and A. Fabrocini, Phys. Rev. C 38 (1988) 1010.

[29] A. Akmal, V. R. Pandharipande, and D. G. Ravenhall, Phys. Rev. C 58 (1998) 1804.

[30] H. Lenske and C. Fuchs, Phys. Lett. 345B (1995) 355.

[31] C. Fuchs, H. Lenske, and H. H. Wolter, Phys. Rev. C 52 (1995) 3043.

[32] S. Typel and H. H. Wolter, Nucl. Phys. A656 (1999) 331.

[33] F. Hofmann, C. M. Keil, and H. Lenske, Phys. Rev. C 64 (2001) 034314.

[34] T. Nikšić, D. Vretenar, P.Finelli and P. Ring, Phys. Rev. C 66 (2002) 024306.

[35] S. F. Ban, J. Li, S. Q. Zhang, H. Y. Jia, and J. P. Sang, and J. Meng, Phys. Rev. C 69 (2004) 045805 .

[36] M. Alford, K. Rajagopal, and F. Wilczek, Phys. Lett. 422B (1998) 247.

[37] R. Rapp, T. Schäfer, E. V. Shuryak, and M. Velkovsky, Phys. Rev. Lett. 81 (1998) 53.

[38] M. Buballa, Phys. Rept. 407 (2005) 205.

[39] D. Blaschke, S. Fredriksson, H. Grigorian, A. M. Öztas and F. Sandin, Phys. Rev. D 72 (2005) 065020 .

[40] S. B. Ruster, V. Werth, M. Buballa, I. A. Shovkovy, and D. H. Rischke, Phys. Rev. D 72 (2005) 034004.

[41] H. Abuki and T. Kunihiro, Nucl. Phys. A 768 (2006) 118.

[42] S. Lawley, W. Bentz, and A. W. Thomas, J. Phys. G: Nucl. Part. Phys. 32 (2006) 667.

[43] S. Lawley, W. Bentz, and A. W. Thomas, Phys. Lett. B632 (2006) 495.

[44] P. Wang, S. Lawley, D. B. Leinweber, A. W. Thomas, and A. G. Williams, Phys. Rev. C 72 (2005) 045801.

[45] S. Ray, A. L. Espíndola, M. Malheiro. J. P. S. Lemos, and V. T. Zanchin, Phys. Rev. D 68 (2003) 084004.

[46] N. K. Glendenning, Astrophys. J. 293 (1985) 470.

[47] H. Huber, F. Weber, M. K. Weigel, and Ch. Schaab, Int. J. Mod. Phys. E 7, No. 3 (1998) 301.

[48] J. L. Friedman, J. R. Ipser, and L. Parker, Astrophys. J. 304 (1986) 115.

[49] S. Barshay and G. E. Brown, Phys. Lett. 47B (1973) 107.

[50] G. E. Brown, K. Kubodera, D. Page, and P. Pizzochero, Phys. Rev. D 37 (1988) 2042.

[51] D. B. Kaplan and A. E. Nelson, Phys. Lett. 175B (1986) 57; ibid. Nucl. Phys. A479 (1988) 273.

[52] G. E. Brown, K. Kubodera, and M. Rho, Phys. Lett. 192B (1987) 273. 
[53] Ch. Fuchs, Prog. Part. Nucl. Phys. 56 (2006) 1.

[54] G. E. Brown, Phys. Bl. 53 (1997) 671.

[55] V. Thorsson, M. Prakash, and J. M. Lattimer, Nucl. Phys. A572 (1994) 693.

[56] F.-K. Thielemann, M.-A. Hashimoto, and K. Nomoto, Astrophys. J. 349 (1990) 222.

[57] F. Weber, M. Meixner, R. P. Negreiros, and M. Malheiro, Ultra-Dense Neutron Star Matter, Strange Quark Stars, and the Nuclear Equation of State, (astro-ph/0606093).

[58] R. L. Jaffe, Phys. Lett. 38 (1977) 195.

[59] N. K. Glendenning and J. Schaffner-Bielich, Phys. Rev. C 58 (1998) 1298.

[60] A. Faessler, A. J. Buchmann, M. I. Krivoruchenko, and B. V. Martemyanov, Phys. Lett. 391B (1997) 255 .

[61] A. Faessler, A. J. Buchmann, and M. I. Krivoruchenko, Phys. Rev. C 56 (1997) 1576.

[62] R. Jaffe and F. Wilczek, Phys. Rev. Lett. 91 (2003) 232003.

[63] D. D. Ivanenko and D. F. Kurdgelaidze, Astrophys. 1 (1965) 251.

[64] N. Itoh, Progr. Theor. Phys. 44 (1970) 291.

[65] H. Fritzsch, M. Gell-Mann, and H. Leutwyler, Phys. Lett. 47B (1973) 365.

[66] G. Baym and S. Chin, Phys. Lett. 62B (1976) 241.

[67] B. D. Keister and L. S. Kisslinger, Phys. Lett. 64B (1976) 117.

[68] G. Chapline and M. Nauenberg, Phys. Rev. D 16 (1977) 450.

[69] W. B. Fechner and P. C. Joss, Nature 274 (1978) 347.

[70] G. Chapline and M. Nauenberg, Ann. New York Academy of Sci. 302 (1977) 191.

[71] N. K. Glendenning, Phys. Rev. D 46 (1992) 1274.

[72] E. Chubarian, H. Grigorian, G. Poghosyan, and D. Blaschke, Astron. \& Astrophys. 357 (2000) 968.

[73] K. Rajagopal and F. Wilczek, The Condensed Matter Physics of QCD, At the Frontier of Particle Physics / Handbook of QCD, ed. M. Shifman, (World Scientific) (2001).

[74] M. Alford, Ann. Rev. Nucl. Part. Sci. 51 (2001) 131.

[75] R. Rapp, T. Schäfer, E. V. Shuryak, and M. Velkovsky, Phys. Rev. Lett. 81 (1998) 53; Ann. Phys. $280(2000) 35$.

[76] K. Rajagopal and F. Wilczek, Phys. Rev. Lett. 86 (2001) 3492.

[77] M. Alford, K. Rajagopal, and F. Wilczek, Nucl. Phys. B537 (1999) 443.

[78] D. T. Son, Phys. Rev. D D59 (1999) 094019. 
[79] M. Alford and S. Reddy, Phys. Rev. D 67 (2003) 074024.

[80] M. Alford, J. Phys. G 30 (2004) S441.

[81] M. Alford, J. A. Bowers, and K. Rajagopal, Phys. Rev. D 63 (2001) 074016.

[82] K. Rajagopal, Acta Physica Polonica B 31 (2000) 3021.

[83] M. Alford, J. A. Bowers, and K. Rajagopal, J. Phys. G 27 (2001) 541.

[84] D. Blaschke, D. M. Sedrakian, and K. M. Shahabasyan, Astron. \& Astrophys. 350 (1999) L47.

[85] C. Alcock, E. Farhi, and A. V. Olinto, Astrophys. J. 310 (1986) 261.

[86] J. Madsen, Lecture Notes in Physics 516 (1999) 162.

[87] M. Alford, K. Rajagopal, S. Reddy, and A. W. Steiner, Phys. Rev. D 73 (2006) 114016.

[88] M. Stejner and J. Madsen, Phys. Rev. D 72 (2005) 123005.

[89] Ch. Kettner, F. Weber, M. K. Weigel, and N. K. Glendenning, Phys. Rev. D 51 (1995) 1440.

[90] N. K. Glendenning, Ch. Kettner, and F. Weber, Phys. Rev. Lett. 74 (1995) 3519.

[91] G. J. Mathews, I.-S. Suh, B. O’Gorman, N. Q. Lan, W. Zech, K. Otsuki, and F. Weber, J. Phys. G: Nucl. Part. Phys. 32 (2006) 1.

[92] V. V. Usov, Phys. Rev. Lett. 80 (1998) 230.

[93] V. V. Usov, Astrophys. J. 550 (2001) L179.

[94] V. V. Usov, Astrophys. J. 559 (2001) L137.

[95] K. S. Cheng and T. Harko, Astrophys. J. 596 (2003) 451.

[96] C. Vogt, R. Rapp, and R. Ouyed, Nucl. Phys. A735 (2004) 543.

[97] K. Thorne, Astrophys. J. 212 (1977) 825.

[98] D. G. Yakovlev and C. J. Pethick, Ann. Rev. Astron. Astrophys. 42 (2004) 169.

[99] L. D. Landau and E. M. Lifshitz, Statistical physics, 3rd ed. (Pergamon Press, Oxford, 1980).

[100] D. G Yakovlev, K. P. Levenfish, and Y. A. Shibanov, Uspekhi Fizicheskikh Nauk 42 (1999) 737, (astro-ph/9906456).

[101] H. Heiselberg, C. J. Pethick, and E. F. Staubo, Phys. Rev. Lett. 70 (1993) 1355.

[102] T. Endo, T. Maruyama, S. Chiba, and T. Tatsumi, Prog. Theor. Phys. 115 (2006) 337.

[103] M. B. Christiansen and N. K. Glendenning, Phys. Rev. C 56 (1997) 2858.

[104] R. Fernández and A. Reisenegger, Astrophys. J. 625 (2005) 291.

[105] A. D. Kaminker, D. G. Yakovlev, A. Y. Potekhin, N. Shibazaki, P. S. Shternin, nad O. Y. Gnedin, Mon. Not. Roy. Astron. Soc. 371 (2006) 477.

[106] N. K. Glendenning and S. Pei, Phys. Rev. C 52 (1995) 2250.

[107] J. L. Zdunik, M. Bejger, P. Haensel, and E. Gourgoulhon, Astron. \& Astrophys. 450 (2006) 747. 